Supplement of

\title{
A mass-weighted atmospheric isentropic coordinate for mapping chemical tracers and computing inventories
}

Yuming Jin et al. 


\section{S1 Computation of equivalent potential temperature $\left(\theta_{\mathrm{e}}\right)$ and dry air mass $(\mathrm{M})$ of atmospheric field}

Saturation vapor pressure $\left(\mathrm{P}_{\mathrm{S}, \mathrm{V}}\right.$, mbar $)$ is computed following:

$$
P_{S, V}=0.06122 \cdot e^{\frac{17.67 \cdot T}{T+243.5}}
$$

where $\mathrm{T}$ is the temperature of air in unit of ${ }^{\circ} \mathrm{C}$.

Water vapor mixing ratio ( $\mathrm{w}, \mathrm{kg}$ water vapor per $\mathrm{kg}$ dry air mass) is computed following Bolton (1980) as:

$$
\mathrm{w}=\mathrm{RH} \cdot 0.622 \cdot \frac{\mathrm{P}_{\mathrm{s}, \mathrm{v}}}{\mathrm{P}-\mathrm{P}_{\mathrm{s}, \mathrm{v}}}
$$

where $\mathrm{RH}$ is relative humidity in $\mathrm{kg} \mathrm{kg}^{-1}$, and $\mathrm{P}$ is barometric pressure in mbar.

Equivalent potential temperature, $\theta_{\mathrm{e}}$, is computed following Stull (2012) as:

$$
\theta_{e}=\left(T+\frac{L_{v}(T)}{C_{p d}} \cdot w\right) \cdot\left(\frac{P_{0}}{P}\right)^{\frac{R_{d}}{C_{p d}}}
$$

where $\mathrm{T}(\mathrm{K})$ is the temperature of air, $\mathrm{R}_{\mathrm{d}}\left(287.04, \mathrm{~J} \mathrm{~kg}^{-1} \mathrm{~K}^{-1}\right)$ is the gas constant for air, $\mathrm{C}_{\mathrm{pd}}\left(1005.7 \mathrm{~J} \mathrm{~kg}^{-1} \mathrm{~K}^{-1}\right)$ is the specific heat of dry air at constant pressure, $\mathrm{P}_{0}\left(1013.25\right.$, mbar) is the reference pressure at the surface, and $\mathrm{L}_{\mathrm{V}}(\mathrm{T})$ is the latent heat of evaporation at temperature $\mathrm{T}$. $\mathrm{L}_{\mathrm{v}}(\mathrm{T})$ is defined as $2406 \mathrm{~kJ} \mathrm{~kg}^{-1}$ at $40{ }^{\circ} \mathrm{C}$, and $2501 \mathrm{~kJ} \mathrm{~kg}^{-1}$ at $0{ }^{\circ} \mathrm{C}$ and scales linearly with temperature.

The mass of air $(\mathrm{M}, \mathrm{kg})$ for each grid cell $\mathrm{x}$ is then calculated by using pressure $(\mathrm{P})$, latitude $(\Phi)$, longitude $(\lambda)$, radius of the earth $(\mathrm{R}, 6371 \mathrm{~km})$ and gravity constant $(\mathrm{g})$ following

$$
\mathrm{M}_{\mathrm{x}}=\frac{\Delta \mathrm{P}}{\mathrm{g}} \cdot|\Delta \sin (\Phi) \cdot \Delta \lambda| \cdot \mathrm{R}^{2}
$$

where $\Delta$ represents the difference between two boundaries of each grid cell.

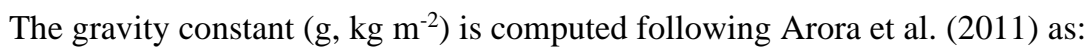

$$
g(\text { lat, } p)=g_{0} \cdot\left(1+0.0053 \cdot \sin ^{2}(\text { lat })-0.000006 \cdot \sin ^{2}(2 \cdot \text { lat })\right)-0.000003086 \cdot h
$$

where the reference gravity constant $\left(\mathrm{g}_{0}\right)$ is assumed to be $9.78046 \mathrm{~m} \mathrm{~s}^{-2}$, and the height (h) in unit of $\mathrm{m}$ is computed from

$$
\mathrm{P}=\mathrm{P}_{0} \cdot \mathrm{e}^{-\frac{\mathrm{h}}{\mathrm{H}}}
$$

where $\mathrm{H}$ is the scale height of the atmosphere and assumed to be $8400 \mathrm{~m}$. 


\section{S2: Contribution of each heating term to the overall time variation of $M_{\theta e}$}

The fractional contributions from different heating terms to the temporal variation of $\mathrm{M}_{\theta \mathrm{e}}$ on seasonal and synoptic scales are computed by using a vector projection method (Graven et al., 2013). In this method, each heating term

$35\left(\frac{\partial}{\partial t} M_{\theta_{e}}^{i}\left(\theta_{e}, t\right)\right)$ is projected onto the sum of all the heating terms $\left(\frac{\partial}{\partial t} M_{\theta_{e}}\left(\theta_{e}, t\right)\right)$ via:

$$
\mathrm{x}_{\mathrm{i}}=\frac{\sum_{\mathrm{t}}\left[\frac{\partial}{\partial \mathrm{t}} \mathrm{M}_{\theta_{\mathrm{e}}}^{\mathrm{i}}\left(\theta_{\mathrm{e}}, \mathrm{t}\right) \cdot \frac{\partial}{\partial \mathrm{t}} \mathrm{M}_{\theta_{\mathrm{e}}}\left(\theta_{\mathrm{e}}, \mathrm{t}\right)\right]}{\sum_{\mathrm{t}}\left[\frac{\partial}{\partial \mathrm{t}} \mathrm{M}_{\theta_{\mathrm{e}}}\left(\theta_{\mathrm{e}}, \mathrm{t}\right) \cdot \frac{\partial}{\partial \mathrm{t}} \mathrm{M}_{\theta_{\mathrm{e}}}\left(\theta_{\mathrm{e}}, \mathrm{t}\right)\right]}
$$

with

$$
\frac{\partial}{\partial \mathrm{t}} \mathrm{M}_{\theta_{\mathrm{e}}}\left(\theta_{\mathrm{e}}, \mathrm{t}\right)=\sum_{i} \frac{\partial}{\partial \mathrm{t}} \mathrm{M}_{\theta_{\mathrm{e}}}^{\mathrm{i}}\left(\theta_{\mathrm{e}}, \mathrm{t}\right)
$$

where the sum is over all time steps, and the mean of each $\frac{\partial}{\partial t} M_{\theta_{e}}^{i}\left(\theta_{e}, t\right)$ has been pre-subtracted (i.e.,

$\left.40 \quad \sum_{t} \frac{\partial}{\partial \mathrm{t}} \mathrm{M}_{\theta_{\mathrm{e}}}^{\mathrm{i}}\left(\theta_{\mathrm{e}}, \mathrm{t}\right)=0\right)$. The sum over $\mathrm{x}_{\mathrm{i}}$ equals 1 , but individual $\mathrm{x}_{\mathrm{i}}$ can be either positive or negative and the absolute value can be either larger or smaller than 1 . 
Table S1: Number of data points of each airborne campaign transect for each simulation

\begin{tabular}{c|cccccccccc}
\hline $\begin{array}{c}\text { Airborne } \\
\text { Transect }\end{array}$ & Original & $\begin{array}{c}\text { Equator } \\
\text { to } 30^{\circ} \mathrm{N}\end{array}$ & $\begin{array}{c}\text { Poleward } \\
\text { of } 30^{\circ} \mathrm{N}\end{array}$ & $\begin{array}{c}\text { Surface }- \\
600 \mathrm{mbar}\end{array}$ & $\begin{array}{c}600 \mathrm{mbar} \\
- \text { Trop. }\end{array}$ & $\begin{array}{c}\text { Pacific } \\
\text { Only }\end{array}$ & $\begin{array}{c}\text { Medusa } \\
\text { Coverage }\end{array}$ & $\begin{array}{c}\text { Random } \\
10 \%\end{array}$ & $\begin{array}{c}\text { Random } \\
5 \%\end{array}$ & $\begin{array}{c}\text { Random } \\
1 \%\end{array}$ \\
\hline HIPPO1 SB & 4837 & 1454 & 3383 & 1794 & 3043 & 4837 & 76 & 484 & 242 & 48 \\
HIPPO2 SB & 4665 & 1510 & 3155 & 1945 & 2720 & 4665 & 82 & 451 & 233 & 45 \\
HIPPO2 NB & 5508 & 2428 & 3080 & 2159 & 3349 & 5508 & 93 & 543 & 275 & 54 \\
HIPPO3 SB & 4439 & 1371 & 3068 & 2038 & 2401 & 4439 & 88 & 427 & 222 & 43 \\
HIPPO3 NB & 4086 & 1135 & 2951 & 1790 & 2296 & 4086 & 84 & 399 & 204 & 40 \\
HIPPO4 SB & 5491 & 1602 & 3889 & 2340 & 3151 & 5491 & 81 & 534 & 275 & 53 \\
HIPPO4 NB & 6411 & 3134 & 3277 & 3142 & 3269 & 6411 & 124 & 626 & 321 & 63 \\
HIPPO5 SB & 5538 & 1678 & 3860 & 2569 & 2969 & 5538 & 78 & 548 & 277 & 55 \\
HIPPO5 NB & 4715 & 1705 & 3010 & 2066 & 2649 & 4715 & 86 & 392 & 236 & 39 \\
ATom1 SB & 9832 & 2333 & 7499 & 3186 & 6646 & 9832 & 83 & 455 & 492 & 46 \\
ATom1 NB & 10685 & 3186 & 7499 & 3665 & 7020 & 0 & 59 & 893 & 534 & 89 \\
ATom2 SB & 11372 & 3909 & 7463 & 4057 & 7315 & 11372 & 84 & 1109 & 569 & 111 \\
ATom2 NB & 10741 & 3284 & 7457 & 3792 & 6949 & 0 & 91 & 1042 & 537 & 104 \\
ATom3 SB & 15143 & 3751 & 11392 & 4817 & 10326 & 15143 & 87 & 1460 & 757 & 146 \\
ATom3 NB & 14039 & 4173 & 9866 & 4764 & 9275 & 0 & 92 & 1362 & 702 & 136 \\
ATom4 SB & 13554 & 3683 & 9871 & 5249 & 8305 & 13554 & 84 & 1327 & 678 & 132 \\
ATom4 NB & 11995 & 3626 & 8369 & 4130 & 7865 & 0 & 89 & 1187 & 600 & 119 \\
\hline
\end{tabular}


Arora, K., Cazenave, A., Engdahl, E. R., Kind, R., Manglik, A., Roy, S., Sain, K. and Uyeda, S.: Encyclopedia of solid earth geophysics, Springer Science \& Business Media, 2011.

Bolton, D.: The computation of equivalent potential temperature, Mon. Weather Rev., 108(7), 1046-1053, doi:10.1175/1520-0493(1980)108<1046:TCOEPT>2.0.CO;2, 1980.

50 Graven, H. D., Keeling, R. F., Piper, S. C., Patra, P. K., Stephens, B. B., Wofsy, S. C., Welp, L. R., Sweeney, C., Tans., P. P., Kelley, J. J., Daube, B. C., Kort, E. A., Santoni, G. W. and Bent, J. D.: Enhanced seasonal exchange of $\mathrm{CO}_{2}$ by Northern ecosystems since 1960, Science, 341(6150), 1085-1089, doi:10.1126/science.1239207, 2013.

Stull, R. B.: An introduction to boundary layer meteorology, Springer Science \& Business Media, 2012. 